\title{
Social media and suicide in social movements: a case study in Hong Kong
}

\author{
Paul S. F. Yip ${ }^{1,2} \cdot$ Edward Pinkney $^{1}$ \\ Received: 28 January 2022 / Accepted: 1 February 2022 / Published online: 1 March 2022 \\ (c) The Author(s), under exclusive licence to Springer Nature Singapore Pte Ltd. 2022
}

\begin{abstract}
Research has indicated that excessive and sensationalized suicide reporting can lead to copycat suicides, especially when deaths involve well-known people. Little is known, however, about the impact of the reporting of suspected protestor suicide deaths during social unrest, particularly in an age of social media. In June 2019, the most substantial social unrest in Hong Kong since its handover in 1997 was triggered by the proposed Anti-Extradition Law Amendment Bill (Anti-ELAB). The social unrest subsided when Hong Kong and many parts of the world were hit by Covid-19 and very strict quarantine measures were imposed on crowd gatherings in Hong Kong at the end of January 2020. A number of reported suicides and deaths of undetermined cause took place during this 8-month period that received considerable attention. To better understand the possible effects of these highly publicized deaths, we examined media reports of suspected suicide cases before, during and after the protest period, as well as topics of suicide-related threads and their replies in social media forums. We found no clear evidence of increased rates of suicide as a result of these incidents, or during the protest period; however, it is suggested that certain narratives and attention surrounding the suspected suicides and undetermined deaths may have contributed to collective emotions such as sadness and anxiety. Some implications for misinformation (intentionally or un-intentionally) and mitigation of suicide risk during social unrest are discussed.
\end{abstract}

Keywords Social movement $\cdot$ Suicide reporting $\cdot$ Suicide $\cdot$ Social media $\cdot$ Protest

Paul S. F. Yip

sfpyip@hku.hk

1 Centre for Suicide Research and Prevention, The University of Hong Kong, Pokfulam, Hong Kong

2 Department of Social Work and Social Administration, The University of Hong Kong, Pokfulam, Hong Kong 


\section{Introduction}

The use of social media has for many become an indispensable part of everyday life, offering access to news, insights, and social interaction. On average users spend almost $2 \mathrm{~h}$ a day interacting with social media platforms [1], and their relatively decentralized form has made them a useful tool during emergencies and natural disasters, helping provide real-time updates, allocate resources and organize volunteers [2]. Social media has also been used by many social activists as an effective platform for information exchange and coordination in social movement worldwide during the past decade [3] and has been identified as a key method of mobilizing participants in Hong Kong's Umbrella Movement protests in 2014 [4] and Anti-Extradition Law Amendment Bill (Anti-ELAB) protests in 2019 [5].

As an open forum, social media allows researchers to see the "digital footprint" of political participation, which is essential to understanding the dynamics of modern-day activism, particularly when "leaderless" or self-organized and uncoordinated political actions are commonplace [3]. Despite the utility of social media during certain events and incidents, there are concerns about how information may be misrepresented (intentionally or unintentionally), and the impact on social behaviours and mental health. The spread of "fake news" has been a concern, including surrounding political events such as the US election of 2016, and the risks of misinformation over social media have been described by the World Economic Forum as one of the major global threats to society [6]. Evidence shows that the demographic characteristics of the deceased person may influence emotional responses, and some emotional responses like sadness and anger show positive correlation with following days' suicide count [27]. Meanwhile, certain forms of reporting of suicide in the media have been shown to increase the suicide rate $[7,8]$, and there is evidence that social media engagement with prominent suicides may also increase incidence of suicide [9].

During the period June 2019-January 2020, Hong Kong saw the largest series of protests in recent history, triggered by the proposed Anti-Extradition Law Amendment Bill (Anti-ELAB). Mass marches and peaceful rallies were interspersed with increasingly confrontational stand-offs over the following 6 months, culminating in fiery street clashes between police and demonstrators across the city, including in the central business districts and at a number of universities in November 2019. It is estimated that more than 16,000 rounds of tear gas were fired across the 6-month period.

From January 2020, the frequency of protest gatherings began to subside with the emergence of COVID-19, after which very strict social distancing measures have been in place. During the period of social unrest, nearly 10,000 protesters, mainly young people, had been arrested and many were charged with serious offences such as rioting.

Over the protest period, several suspected protest-related suicides were widely reported. In particular, the death of a 35-year-old man (Mr Leung Ling-kit) with an Anti-ELAB banner who, despite the efforts of firemen, policemen and onlookers, fell from a building in Admiralty, Hong Kong, gained considerable 


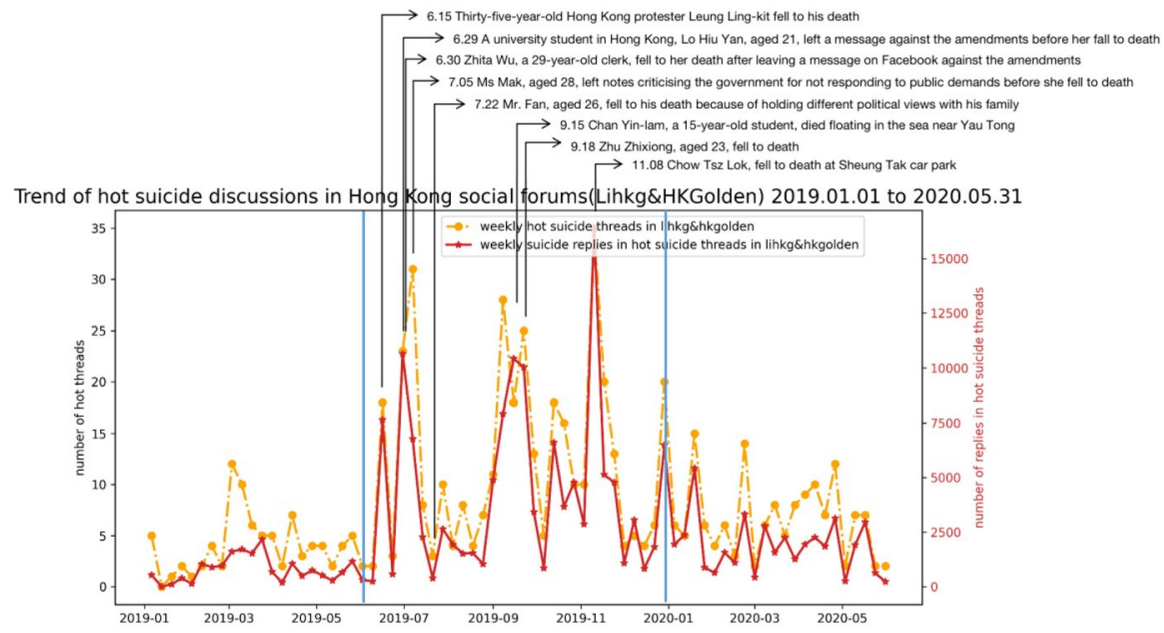

Fig. 1 Trend of hot suicide discussions (threads and replies) in Hong Kong social forums (Lihkg \& HKGolden) from January 1, 2019 to May 31, 2020. Suspected suicide cases that are related to the AntiELAB protest reported were marked on the corresponding time point

public attention. Tribute art portraying the yellow raincoat worn by Mr. Leung was widely circulated and there was much discussion. Further suspected suicides followed and were linked to the protest movement by media reports and online discussion. Figure 1 shows the trend of social media hot threads (top 10\% threads with most replies) during January 2019-May 2020, where the discussion peaks usually followed those suspected suicides and/or related deaths.

Remembrance gatherings related to the deaths and other forms of recognition of these cases were presented as an opportunity for cathartic collective grieving and sense-making of the incident as part of wider concerns about the government bill, and such gatherings may have conceivably offered comfort to loved ones, as well as encouraging social support and help-seeking behaviours for those that may have been feeling desperate, potentially contributing to a positive social cohesion effect [10]. On the other hand, there were concerns that the attention around these deaths and possible subsequent romanticisation may contribute to overly sensationalized and dramatized reporting, leading to increased suicidal risk amongst those who may be unstable and vulnerable, or copycat suicides of the kind found by researchers to occur after highly reported suicide deaths of famous people (known as the "Werther Effect") [7, 8, 11, 12].

Additional suspected suicides and other undetermined deaths of protesters that were reported online and discussed in social media occurred in subsequent days, weeks and months, coinciding with the most intense period of the protests. Following an incident between police and protesters at a Hong Kong subway station in late August 2019, rumours of deaths being covered up or suppressed to avoid political consequences increased, and speculation about possible foul play relating to two particular deaths in September and November ensued, including that evidence was concealed [13]. These were the deaths of Ms. Chan Yin-lam (aged 15, a student, 
found unclothed at sea) and Mr Chow Tsz-lok (aged 22, a student that fell from a height in a carpark compound). Both deaths stirred considerable media suspicion, emotion and rumours of possible foul play. Some protesters even took to social media to share videos or photos in which they stated categorically that they would not take their own lives, in order to address fears involving suspicions and mistrust, should anything happen to them [14]. It turns out the two cases have been given the verdict of underdetermined cause of death for Ms. Chan and an open verdict to $\mathrm{Mr}$ Chow death by the Coroner Court recently. Unhelpful and sensational speculation (intentionally and/or unintentionally) may have been avoided had these cases been investigated by the Coroner Court more urgently.

It is well established that suicide is a complex phenomenon generally resulting from many factors [15]. However, a desire to attribute a suspected suicide to specific causes may be particularly strong in certain political circumstances, and contribute to perceptions of suicide as a form of political martyrdom, or expression of collective frustrations or despair over perceived social injustice, stirring up the emotions in the community to provide the spark or sustenance for a social movement, such as in the case of Thich Quang Duc in Vietnam in 1963 [16], Ryszard Siwiec in Warsaw in 1968 [17], Jan Palach in Prague in 1969 [18], and Mohamed Bouazizi prior to the Arab Spring protests in 2010 [19]. This raises questions over how these deaths in Hong Kong may have influenced the ensuing escalation in social unrest.

Suicide prevention professionals and media operators can find themselves in a difficult position. On the one hand, they have a duty to mitigate the risk of suicide contagion, a phenomenon that has been seen also in protest-related suicides [20]; on the other hand, where there are major social tensions, journalists may be concerned about appearing to suppress coverage of acts that could be perceived as political statements, or of undermining freedom of speech [21]. This was a situation that Hong Kong found itself in during the 2019 protests, as suspected suicides became linked in the media, not only locally but also internationally, to themes of martyrdom and self-sacrifice [23-25]. Such portrayals are discouraged by the best practice guidelines of suicide reporting due to concerns of the possibility of similar copycat incidents, as per the Werther Effect [25].

Evidence linking media reporting on suicide with suicide cases has led to media being urged to avoid sensationalizing cases or giving too much detail [25], with some experts suggesting reducing the coverage of suicides altogether [26]. There is evidence that the quantity of social media responses to suicide deaths, and the sentiment characterized by those responses, may predict subsequent incidence of suicide $[9,27]$. This may be especially pertinent during times of political upheaval or social unrest, when citizen journalists or activists may use media to stimulate emotions or conversations. At present, there is little research into how existing media guidelines and suppression of suicide stories may or may not lead to discussions being pushed underground into social media channels, at risk of further stirring speculation and reliance on unauthoritative source materials. Good media management for suicide prevention must consider political contexts and secondary effects beyond traditional media.

One important consideration for understanding the social movement and suicide contagion risks involves the mechanisms by which social media may have 
propagated narratives and speculation relating to these deaths. In this paper, first, we examine the patterning of suspected suicides and deaths with undetermined causes, and the level of engagement and discussion they created in social media. While these protest deaths are not the sort of "celebrity deaths" that have been well studied for copycat effects, it is suggested that the impact on vulnerable individuals could be as much if not more due to the perception that they were fellow members of the protest movement and, therefore, highly relatable. This paper, second, looks at the evidence for an increase in suicide rate during the protest period.

By analyzing social media data and the characteristics and phenomena of social media discussion on suicide in this article, we also consider whether these deaths and the beliefs around them, including speculation that the deaths involved acts of self-sacrifice or foul play, may have been the catalyst for increased social media engagement and provided an emotional anchor to unite protesters together, possibly romanticizing the deaths and offering impetus to sustain the social movement through the end of 2019 .

\section{Data and methods}

\section{Stage definitions}

For the purpose of our study as well as the ISO week date standard, we define the Pre-protest period as from January 1 to June 2, 2019, the Peri-protest period as June 3 to December 29, 2019, and the Post-protest (COVID) period as from December 30, 2019, to May 31, 2020. While sporadic protests persisted until June 2020, these were less conspicuous than during the second half of 2019.

\section{Suicide cases}

The media reporting rate of suicides in Hong Kong has been found to be much higher than that of western countries $[8,28,29]$ of actual cases matched against coroners' rulings, enabling reports to serve as an early advanced estimate of number of cases as it usually takes more than 6 months for the Coroner to reach a verdict [30]. We collected the daily suicide cases reported by news outlets in Hong Kong from the database of Meltwater (See Appendix 1). Suicide-related keywords (Appendix 2 for Original Cantonese and English translation version) were used as the primary filters for collecting the information of suicide cases. After that, two well-trained coders coded each suicide news items independently, including removing non-suicide news, identifying duplicates, and coding suicide related features. For each case, the coders marked the suicide method, date, gender, age, location, and possible reasons mentioned in the news. Disagreements were discussed and resolved among two coders. After the data cleaning, there was a total of 1013 unique suicide cases reported during the study period (January 2019-May 2020). 


\section{Social media data}

Social forum discussions about suicide-related topics were aggregated for trend and sentiment analysis. The two popular forums, LIHKG and Golden, were our target forums as they were widely used by Hong Kong local people and AntiELAB movement supporters [31, 32]. We systematically crawled all suiciderelated threads and replies to those threads from the above social forums from January 12019 to May 312020 using the API provided by the service providers. In the social forum context, each new discussion started is called a thread and the subsequent replies are the number of replies arising from the thread. The thread can be started by anyone who can post in the forum. Likewise, the number of replies can be linked to the particular thread. We define suicide-related threads as the threads in which the user mentioned any suicide-related keywords, such as "suicide", "jumping from roof", "hanging" (for the full list and its original Cantonese version, see Appendix 2). In order to understand the surges and peaks in the Peri-protest period and other periods, we introduce the "hot thread" as one of the measurements. Hot threads are defined as the top $10 \%$ threads with most replies.

\section{Two popular narratives}

We apply a list of keywords that indicate the perception of martyrdom (full list see Appendix 3) to all threads and replies. If any reply in one thread mentioned any keyword in the list, this thread will be categorized as a martyrdom mentioning thread. If any reply mentioned any keywords in the list, this reply will be categorized as a martyrdom mentioning reply. By the same methods and definition in the previous section, we apply a list of keywords that indicated the perception of foul play including forced suicide by others (full list see Appendix 4) to all threads and replies.

\section{Sentiment analysis}

Social media forums provide a range of stories and information to the public and are used as an important tool of communicating with others during COVID-19 and the protests. Analyzing the sentiment of these widely discussed topics and issues can allow us to infer the emotional climate of the general public as well as events themselves. All text replies were extracted for sentiment analysis with the help of LIWC (Technical details see Appendix 5).

\section{Statistical analysis}

Weekly suicide reported cases in the news were extracted and compared on the three predefined stages, Pre-protest, Peri-protest and Post-protest, in order to get an overview of suicide trends. Weekly suicide threads and replies on social 
forums were extracted and compared between three stages to understand the trend of how people use social forums to discuss suicide and suspect suicide cases and news. We further investigated the thread initiators to establish whether this was a centralized behaviour driven by several super spreaders (KOLs) and/or decentralized behaviour with a wide base of initiators. Last, we compare discussions with narratives versus discussions without narratives on different sentiment categories. ANOVA were used for testing differences among means. Post-hoc pairwise comparisons were performed with Tukey's HSD adjustment if statistically significant ANOVA results were observed. Eta-squared and rank-biserial correlation were computed to calculate the effect size of the results. Considering the distribution of the sentiment score is not normally distributed and there are a lot of zeros, Mann-Whitney $U$ Test are used to test whether distributions of sentiments scores are equal.

\section{Results}

\section{Social media discussions about suicide among the three periods}

In total, 1,244,746 threads were collected, 235,160 of which were from Golden and 1,009,586 from LIHKG. Of these, 1961 (0.83\%) threads from Golden and 4203 $(0.42 \%)$ threads from LIHKG were detected as suicide-related threads. Within these threads, 29,940 replies from Golden and 234,728 replies from LIHKG were collected. Figure $2 \mathrm{a}, \mathrm{b}$ shows the trend of suicide threads and replies during the study period. Both suicide threads and replies in the Peri-protest period are higher than the other two periods. Table 1a shows that significant levels have been observed in suicide threads count by pair-wise comparison between the Peri-protest and the Pre-protest period $(p=0.004)$, the Peri-protest and Post-protest period $(p=0.001)$. Table $1 \mathrm{~b}$ reports a similar pattern in suicide replies comparison, where significant levels have been observed between the Pre and Peri period $(p=0.001)$, the Peri and Post period $(p=0.001)$. However, there is no significant difference between the Pre- and Post- period, regardless of suicide threads and replies.

We also examined the popular threads and who initiated those discussions. 300 (48.5\%) of hot threads are posted by one-time users (the user who post suicide-related threads only once in the study period), they are not necessarily the key opinion leaders (KOL) and 478 (77\%) of hot threads are posted by users with less than five total threads. The results showed a decentralized pattern in initiating the hot thread discussions. This suggests a wide base in the community were participating in discussion, and not only a small number of individuals attempting to spark publicity or to steer conversations. 
$\mathbf{a}$

Trend of suicide threads in Hong Kong social forums(Lihkg\&HKGolden) 2019.01.01 to 2020.05.31

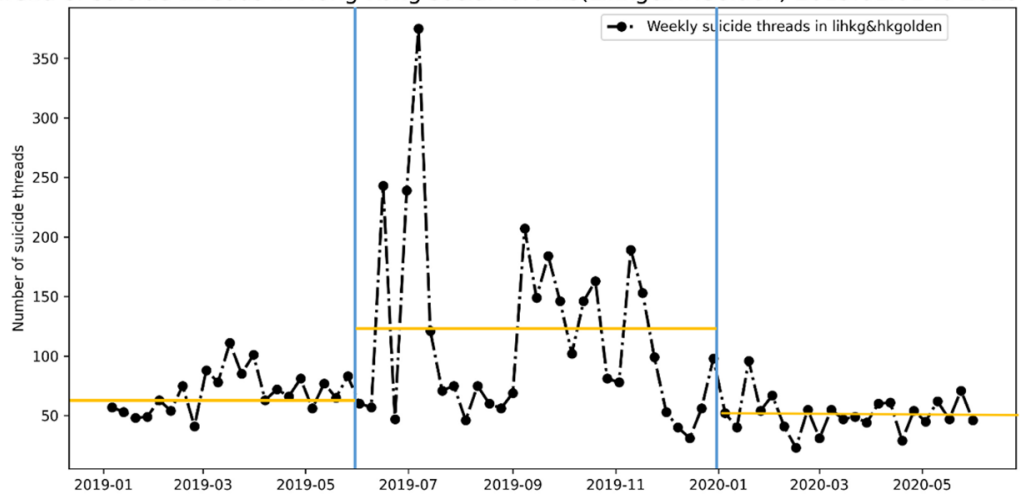

b Trend of suicide replies in Hong Kong social forums(Lihkg\&HKGolden) 2019.01.01 to 2020.05.31

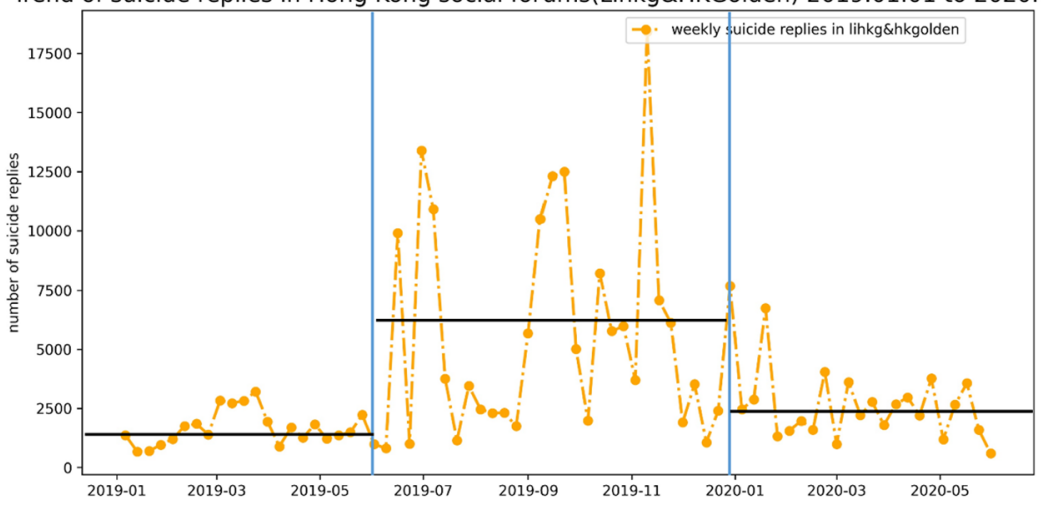

Fig. 2 a Weekly suicide related threads in social forums (Lihkg \& HKGolden) from January 2019 to May 2020. Yellow line in the figure represents the mean of weekly suicide threads from social forums. The number is $69(\mathrm{SD}=18)$ in the Pre-protest period, $117(\mathrm{SD}=78)$ in the Peri-protest period, and 51 $(\mathrm{SD}=16)$ in the Post-protest (COVID) period. b Weekly replies on suicide related threads in social forums (Lihkg \& HKGolden) from January 2019 to May 2020. Black line in the figure represents the mean of weekly replies in suicide threads from social forums. The number is $1646(\mathrm{SD}=724)$ in the Pre-protest period, $5752(\mathrm{SD}=4485)$ in the Peri-protest period, and $2499(\mathrm{SD}=1335)$ in the Post-protect (COVID) period

\section{Suicide cases reported among the three periods}

Figure 3 reports the trend of suicide reported cases in the study period. The results showed that, contrary to some expectations, the total (or mean weekly) suicides in the protest period were actually slightly lower than the Pre- and Peri-protest periods. Multi-comparisons results in Table 2 revealed that there is no significant change in the suicide incidence based on the news reporting among the three periods. This does not mean that there was no copycat effect in individual cases or escalated suicide atmosphere. The high volatility in the weekly cases leaves open the question of whether there is some cancellation effect among the population, i.e., some groups 
Table 1 Comparisons of Means of (a) weekly suicide threads in social forums on three periods, (b) weekly replies of suicide threads in social forums on three periods

\begin{tabular}{llrrrrrr}
\hline Periods & $N$ (weeks) & \multicolumn{1}{c}{ Mean } & \multicolumn{1}{l}{ SD } & \multicolumn{1}{l}{$F$} & $p$-value & $\eta^{2}$ & Tukey's HSD \\
\hline (a) & & & & & & & \\
Pre-protest (I) & 22 & 69.36 & 17.63 & 11.59 & 0.000 & 0.246 & II $>$ I = III \\
Peri-protest (II) & 30 & 116.97 & 77.58 & & & & \\
Post-protest (III) & 22 & 51.32 & 15.53 & & & & \\
(b) & & & & & & & \\
Pre-protest (I) & 22 & 1655.46 & 724.99 & 14.02 & 0.000 & 0.283 & II $>$ I $=$ III \\
Peri-protest (II) & 30 & 5767.80 & 4493.05 & & & & \\
Post-protest (III) & 22 & 2509.73 & 1336.15 & & & & \\
\hline
\end{tabular}

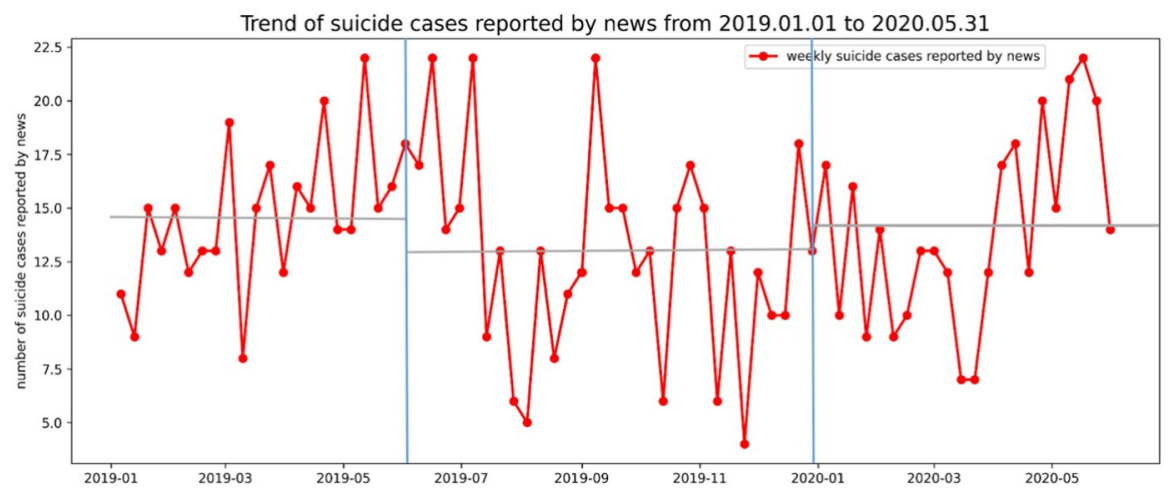

Fig. 3 Trend of suicide cases reported by news in Hong Kong from January 2019 to May 2020. Grey line in the figure represents the mean of weekly suicide cases reported by news in three periods. The number is $14.6(\mathrm{SD}=3.3)$ in the Pre-protest period, $12.8(\mathrm{SD}=4.8)$ in the Peri-protest period, and 14.0 $(\mathrm{SD}=4.6)$ in the Post-protest (COVID) period

Table 2 Comparisons of Means of weekly suicide cases reported by news in Hong Kong on three periods

\begin{tabular}{lllllll}
\hline Periods & $N$ (weeks) & Mean & SD & $F$ & $p$-value & $\eta^{2}$ \\
\hline Pre-protest & 22 & 14.636 & 3.346 & 1.265 & 0.289 & 0.034 \\
Peri-protest & 30 & 12.767 & 4.833 & & & \\
Post-protest & 22 & 14.000 & 4.461 & & & \\
\hline
\end{tabular}

might experience higher risk whereas the others might have lower risk, and the overall rate might not show any change as shown in the suicide cases during COVID-19 [33]. In the discussion, we consider some of the factors that may lead to lower risk amongst some individuals. 


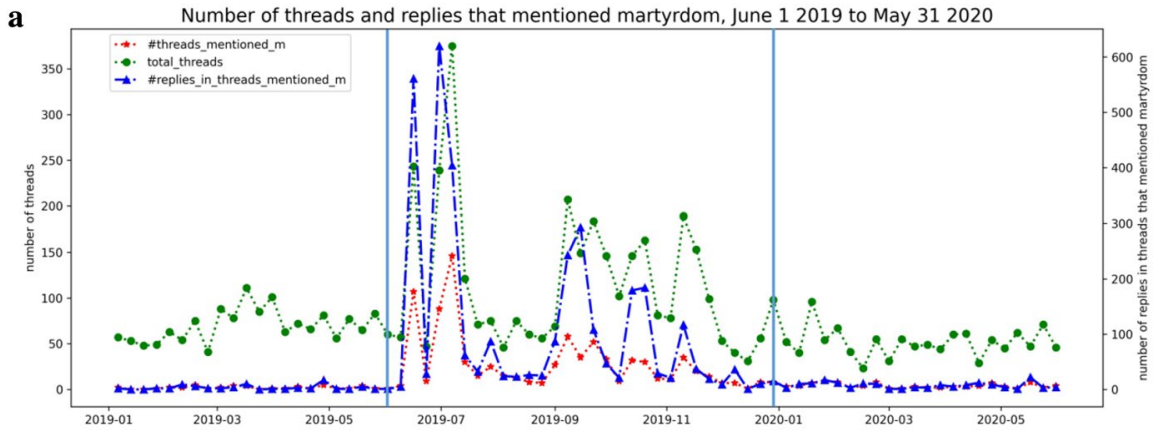

b

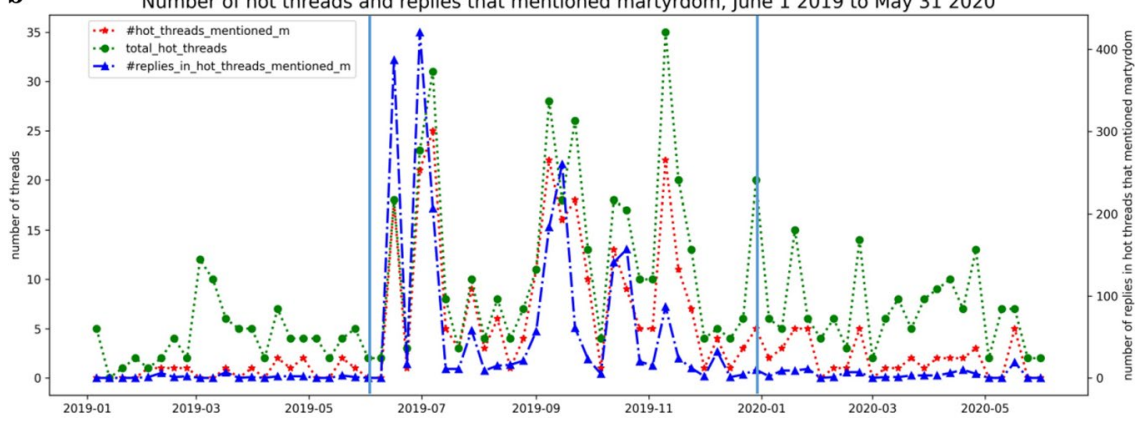

Fig. 4 a Number of threads and replies that mentioned martyrdom narrative in social forums from January 2019 to May 2020. b Number of hot threads and replies that mentioned martyrdom narrative in social forums from January 2019 to May 2020

\section{Narratives that dominated suicide discussions in the protest period}

\section{"Martyrdom"}

Figure 4a shows the trend of total threads, number of threads that mentioned martyrdom and number of replies in those threads that mentioned martyrdom. Figure $4 \mathrm{~b}$ shows the trend for hot threads and their replies. The martyrdom related keywords started to be widely used from June 16 2019, after the first death on June 9. The situation is more substantial in the hot thread discussions, where $68 \%$ of hot threads in the protest period are martyrdom mentioning threads compared to $24 \%$ of total threads are martyrdom mentioning threads. The mentioning phenomenon gradually cooled down when stepping into the COVID period. Table 3 reports sentiment comparisons between martyrdom mentioning posts and no martyrdom mentioning posts. The results reveal that the anxiety score and sadness score of martyrdom mentioning posts are significantly higher than posts without mentioning martyrdom $(U=510,248,952.5, P<0.001$ and $U=506,100,631.5, P<0.001)$. Meanwhile, martyrdom mentioning posts have significantly lower scores of anger and lower overall 
Table 3 Sentiment comparisons between martyrdom mentioning posts and no martyrdom mentioning posts

\begin{tabular}{llllllll}
\hline Emotion categories & $\begin{array}{l}\text { Martyrdom } \\
\text { mentioned }\end{array}$ & $n$ & Mean & SD & $U$-value & $p$-value & $\mathrm{RBC}^{\mathrm{b}}$ \\
\hline Anxiety & False & 260,187 & 0.25 & 2.18 & $510,248,952.5$ & 0.000 & -0.093 \\
& True & 3587 & 0.35 & 1.51 & & & \\
Anger & False & 260,187 & 1.19 & 6.73 & $530,213,054.5$ & 0.000 & -0.136 \\
& True & 3587 & 0.96 & 3.10 & & & \\
Sadness & False & 260,187 & 0.32 & 2.62 & $506,100,631.5$ & 0.000 & -0.085 \\
& True & 3587 & 0.36 & 1.43 & & & \\
\multirow{2}{*}{ OSS $^{\text {c }}$} & False & 260,187 & 0.47 & 0.27 & $410,300,122$ & 0.000 & 0.121 \\
& True & 3587 & 0.42 & 0.31 & & & \\
\hline
\end{tabular}

${ }^{a}$ Mann-Whitney $U$ test value

${ }^{\mathrm{b}}$ Rank-biserial correlation

${ }^{c}$ Overall Sentiment Score, aggregated by both positive score and negative score, See Appendix 5 for more details

sentiment than posts without mentioning martyrdom $(U=530,213,054.5, P<0.001$, $U=410,300,122, P<0.001)$.

\section{"Foul play"}

Figure 5a shows the number of threads and replies that mentioned foul play phenomenon and Fig. 5b shows the number of hot threads and replies that mentioned foul play phenomenon. The first surge of this phenomenon is around the middle of September, 2019, and it peaked at the week of November 4-10 where the death of the university student, Mr. Chow Tsz Lok had been announced. The situation is also more notable in the hot thread discussions, where $40 \%$ of hot threads in protest period mentioned foul play compared to $18 \%$ of total threads mentioned foul play. Unlike martyrdom, the mention of foul play extended into the COVID period. Table 4 reports sentiment comparisons between foul play and no foul play mentioning posts. The results reveal that posts mentioning foul play narrative have higher anxiety score while lower anger score than those without mentions $(U=332,715,633, P<0.001, U=318,018,823.5, P<0.001)$.

\section{Discussion}

The 8-month social unrest in 2019 was unprecedented in Hong Kong, and has become a source of sadness, anxiety, and anger for much of the city, with divisions causing conflicts within families and communities that have led to more polarization in the community. A number of high-profile suspected suicides/deaths were reported, raising concerns about a copycat effect or increase in suicides. 

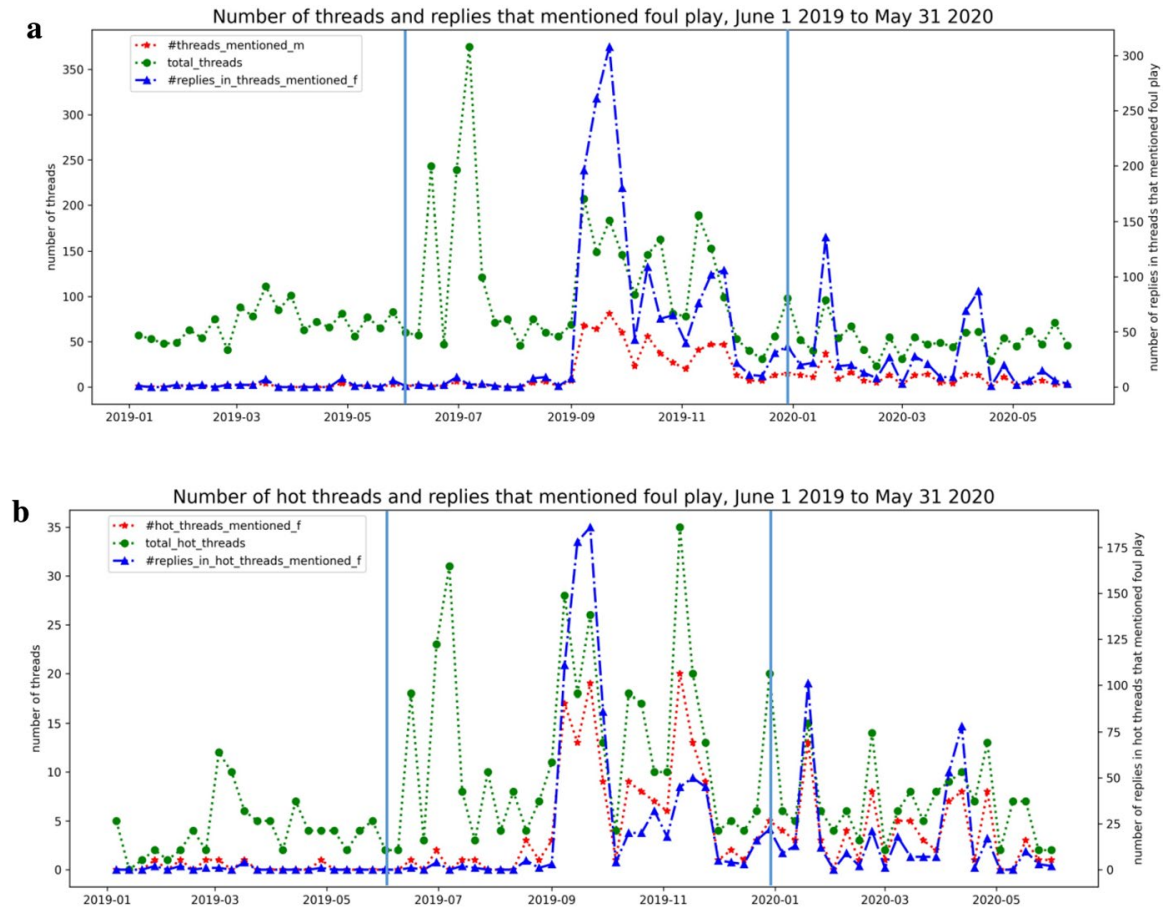

Fig. 5 a Number of threads and replies that mentioned foul play narrative in social forums from January 2019 to May 2020. b Number of hot threads and replies that mentioned foul play narrative in social forums from January 2019 to May 2020

Table 4 Sentiment comparisons between foul play mentioning cases and no foul play mentioning cases

\begin{tabular}{llllllll}
\hline Emotion categories & $\begin{array}{l}\text { Foul play } \\
\text { mentioned }\end{array}$ & $n$ & Mean & SD & $U$-value & $p$-value & RBC \\
\hline Anxiety & False & 261,488 & 0.25 & 2.17 & $332,715,633$ & 0.000 & -0.113 \\
& True & 2286 & 0.57 & 2.07 & & & -0.064 \\
Anger & False & 261,488 & 1.19 & 6.72 & $318,018,823.5$ & 0.000 & -0.009 \\
& True & 2286 & 0.69 & 2.59 & & & 0.020 \\
Sadness & False & 261,488 & 0.32 & 2.62 & $301,448,127.5$ & 0.050 & \\
& True & 2286 & 0.12 & 0.84 & & 0.050 & 0.020 \\
OSS & False & 261,488 & 0.47 & 0.27 & $292,808,739$ & & \\
& True & 2286 & 0.46 & 0.28 & & & \\
\hline
\end{tabular}

Contrary to expectation, we did not find evidence of an increase in suicide rate overall during the protest period when compared to the pre-protest period. However, social media analysis found increased interest and engagement in suicide reporting during the protest period compared to pre-protest and post-protest periods, which could be partially explained by popular or sensationalized narratives-namely those 
pertaining to martyrdom and foul play. The martyrdom narrative in particular exhibited a comparatively negative sentiment, especially for anxiety- and sadness-related language. Such sentiment (intentionally or unintentionally) may have driven further engagement in the social unrest. It also raises concerns from a suicide prevention perspective, suggesting that while the suicide rate did not increase overall, there may have been conditions for copycat or emulated suicides. Given these risks, and also broader features of the protest that may have been conducive to poor mental health such as family conflict, it is perhaps surprising that the suicide rate did not appear to increase during the course of the protests.

The possibly unexpected result may be due to the positive aspects of social gatherings and social cohesion cancelling out negative aspects, suggesting a complicated relationship between suicide and media during periods of social unrest and political tension. Additionally, some sources of support such as counselling and social work assistance were provided and publicised to those affected following the deaths that may have provided alternative means of coping (consistent with the "Papageno Effect") [34]. Thus, while there may have been an increased risk in some, the saliency of the belief that the suicides may have involved protesters may have increased participation in the social movement and given meaning to the lives of some who might not have had the opportunity to participate in mass social gatherings since the Umbrella Movement in 2014. If it is indeed the case that gatherings and social engagement helped to offset some negative effects of heavily reported suicides, then the ongoing suicide rate since the pandemic and related social isolation should be monitored closely.

In order to avoid potential copycat suicides brought by excessive and sensationalized suicide reporting, policymakers and social media operators need to pay attention to early warning signs and be wary of emotional contagion and risk amplification in similar incidents and discussions. Some potential early interventions should be provided, including certain positive cues, fact-checked information and appropriate helpline information. In the long run, a concrete plan needs to be developed to monitor and respond to sensationalized discussions of deaths on social media in a real-time or responsive way to minimize suicide risk. Authorities may also take stronger steps to prevent potential causes for suspicion, for instance, through more timely investigations and Coroner Court inquests into deaths to provide accurate information to the community and counteract disinformation or misinformation [35].

The proliferation of beliefs and suspicions about the motivations of victims and causes of deaths over social media highlights the important role for the media in providing proportionate, factual and reliable information. Authorities should seek to provide open and transparent access to information for media outlets, including those of differing political positions, to assist them with the task of disseminating high quality, rapid information in response to public concerns. Global suicide media guidelines could also be updated to consider complex political factors that can affect reporting, such as during periods of social unrest. Finally, the presence of suicide media reports from international outlets that conflicted with suicide reporting guidelines suggests the need for more globally coordinated standards and monitoring of suicide reporting. Future research may examine this further. 


\section{Limitations}

This study uses media-reported suicide cases as a surrogate (relative indicator) for the actual suicide cases, as the ascertainment of suicide deaths in Hong Kong is determined by Coroner's Court and usually takes at least 6 months or longer to reach the verdict. Hence complete data for the three periods were not available to make a full comparison. Although the reporting of suicides in Hong Kong has been found to be quite extensive and consistent, with $30-40 \%$ of cases being reported in the media, the percentage of cases being reported in the media may have fluctuated over the course of the study period. While there is confidence in the overall trends, further study at a later date may use official figures, which may be used to more precisely examine evidence of copycat suicides. Secondy, although we have used sentiment analysis to allude to possible emotional and behavioural risks, the relationship between sentiments and actual suicide rate remains unknown. Future work can consider conducting real-time sentiment analysis to establish the monitoring of public mood and risk, to further explore the causal relationships between media reports, social media discussions and suicide rate.

\section{Appendix 1}

Meltwater is a software as a service (SaaS) solution and online media monitoring company, which provides both online and printed media data. URL: Meltwater: Media Monitoring \& Social Listening Platform.

\section{Appendix 2}

Suicide related keywords used in collecting suicide news and discussions from Meltwater and social forums.

\begin{tabular}{|c|c|c|c|}
\hline Chinese & English & Chinese & English \\
\hline 自殺 & Suicide & 跳樓 & Jump off from a building \\
\hline 割腕 & Cut wrist & 燒炭 & Charcoal burning \\
\hline 溺水 & Drowning & 吊頸 & Hanging \\
\hline 膠袋笠頭 & $\begin{array}{l}\text { Choking to death with plastic } \\
\text { bag }\end{array}$ & 上吊 & Hanging \\
\hline 仰藥 & Taking drugs & 跳橋 & Jump off from a bridge \\
\hline 輕生 & Commit suicide & 墜軌 & Jump off from a rail \\
\hline 自插 & Self-plug & 墜崖 & Falling off a cliff \\
\hline 自盡 & Suicide & 燒煤氣 & Burning gas \\
\hline 遺書 & Suicide note & 服毒 & Take poison \\
\hline 尋死 & Seek death & 自焚 & Self-immolation \\
\hline 室息 & Suffocation & 割喉 & Cut throat \\
\hline 自縊 ～～ & Hanging & 墮海 & Jump into the sea \\
\hline
\end{tabular}




\section{Appendix 3}

Martyrdom related keywords used for filtering related posts.

\begin{tabular}{ll}
\hline Chinese & English \\
\hline 烈士 & Martyr \\
義士 & Righteous \\
爲香港而死 & Die for Hong Kong \\
捨命 & Sacrifice oneself \\
特牲者 & Martyr \\
特牲 & Sacrifice \\
英雄 & Hero \\
\hline
\end{tabular}

\section{Appendix 4}

Foul play related keywords used for filtering related posts.

\begin{tabular}{ll}
\hline Chinese & English \\
\hline 強迫自殺 & Forced suicide \\
被殺 & Killed \\
謀殺 & Murdered \\
暗殺 & Assassinated \\
非自殺 & Fake suicide \\
非自然死亡 & Unnatural death \\
冤死 & Unjustly died \\
\hline
\end{tabular}

\section{Appendix 5}

\section{Sentiment analysis method}

We used LIWC software to calculate the sentiment score for each post. LIWC was widely used in relevant sentiment tasks, including suicide risk and emotional distress [36], and emotional dynamics in online social movement [37]. The basic logic behind LIWC is they have created a dictionary about different emotions and topics, and it can count the number of keywords of different categories appeared in the posts/articles and calculated a percentage based on the length of the posts/articles. The score can be used in downstream tasks like classification and correlation analysis. There are five categories of emotions predefined in LIWC, including positive emotion, negative emotion, sad, anger and anxiety. We also created an overall 
sentiment score based on the positive score and negative score to represent the general sentiment of one post. The overall sentiment scores

$$
\text { OSS }=\frac{\text { Positive sentiment score }}{\text { Positive sentiment score }+ \text { Negative sentiment score }},
$$

whereas 0.5 were used to represent the posts with both 0 positive score and 0 negative score. For anxiety, anger and sad, the range of the score is $0-100$, the larger the score, the more tendency towards this emotion whereas the range of overall sentiment score (OSS) is between 0 and 1, and the post is more positive if SSO is closer to 1 , and more negative if closer to 0 . There are two steps to get the sentiment score. First, all messages were segmented into a set of keywords with the help of jieba. ${ }^{1}$ In order to better fit Hong Kong cultural context, colloquial words and local slangs were added in jieba dictionary to optimize the word segmentation. Second, all punctuation, emojis were removed before calculating the emotion score.

Funding None.

Availability of data and material Not applicable.

Code availability Not applicable.

\section{Declarations}

Conflicts of interest/Competing interests The authors declare that they have no known competing financial interests or personal relationships that could have appeared to influence the work reported in this paper.

Ethics approval Not applicable.

Consent to participate Not applicable.

Consent for publication Not applicable.

\section{References}

1. Hruska, J., \& Maresova, P. (2020). Use of Social Media Platforms among Adults in the United States-Behavior on Social Media. Societies. https://doi.org/10.3390/soc10010027

2. Mavrodieva, A. V., \& Shaw, R. (2021). Social media in disaster management. in Media and Disaster Risk Reduction. p. 55-73. https://doi.org/10.1007/978-981-16-0285-6_4

3. Teo, E., \& Fu, K.-W. (2021). A novel systematic approach of constructing protests repertoires from social media: Comparing the roles of organizational and non-organizational actors in social movement. Journal of Computational Social Science. https://doi.org/10.1007/ s42001-021-00101-3

4. Lee, F. L. F., \& Chan, J. M. (2018). Media and protest logics in the digital era: The umbrella movement in Hong Kong. Oxford University Press.

5. Purbrick, M. (2019). A Report of the 2019 Hong Kong Protests. Asian Affairs, 50(4), 465-487. https://doi.org/10.1080/03068374.2019.1672397

\footnotetext{
1 A Python Chinese word segmentation module. https://github.com/fxsjy/jieba
} 
6. WorldEconomicForum. (2013). Digital Wildfires in a Hyperconnected World. https://repor ts. weforum.org/global-risks-2013/risk-case-1/digital-wildfires-in-a-hyperconnected-world/. Accessed 10 June 2021

7. Niederkrotenthaler, T., et al. (2020). Association between suicide reporting in the media and suicide: Systematic review and meta-analysis. BMJ, 368, m575. https://doi.org/10.1136/bmj.m575

8. Yip, P. S. F., et al. (2006). The effects of a celebrity suicide on suicide rates in Hong Kong. Journal of Affective Disorders, 93(1-3), 245-252. https://doi.org/10.1016/j.jad.2006.03.015

9. Ueda, M., et al. (2017). Tweeting celebrity suicides: Users' reaction to prominent suicide deaths on Twitter and subsequent increases in actual suicides. Social Science \& Medicine, 189, 158 166. https://doi.org/10.1016/j.socscimed.2017.06.032

10. Ni, M. Y., et al. (2020). Mental health during and after protests, riots and revolutions: A systematic review. Australian and New Zealand Journal of Psychiatry, 54(3), 232-243. https://doi.org/ $10.1177 / 0004867419899165$

11. Chen, Y. Y., et al. (2014). The impact of a celebrity's suicide on the introduction and establishment of a new method of suicide in South Korea. Archives of Suicide Research, 18(2), 221-226. https://doi.org/10.1080/13811118.2013.824840

12. Ji, N. J., et al. (2014). The impact of indiscriminate media coverage of a celebrity suicide on a society with a high suicide rate: Epidemiological findings on copycat suicides from South Korea. Journal of Affective Disorders, 156, 56-61. https://doi.org/10.1016/j.jad.2013.11.015

13. DimSumDaily. (2020). Police refute rumour that the cremation of 15-year-old Chan Yin-lam was done hastily to conceal evidence. DimSumDaily. https://www.dimsumdaily.hk/police-refuterumour-that-the-cremation-of-15-year-old-chan-yin-lam-was-done-hastily-to-conceal-evidence/. Accessed 10 June 2021.

14. Andersen, S. S. (2019). Hong Kong protesters are declaring 'I Won't Kill Myself' just in case they disappear. https://www.vice.com/en/article/7kzpa4/hong-kong-protesters-declaring-notsuicide. Accessed 10 June 2021.

15. Hawton, K., \& Pirkis, J. (2017). Suicide is a complex problem that requires a range of prevention initiatives and methods of evaluation. British Journal of Psychiatry, 210(6), 381-383. https:// doi.org/10.1192/bjp.bp.116.197459

16. Crosby, K., Rhee, J. O., \& Holland, J. (1977). Suicide by fire: A contemporary method of political protest. International Journal of Social Psychiatry, 23(1), 60-69. https://doi.org/10.1177/ 002076407702300111

17. Stach, S. (2016). An ordinary man, a national hero, a Polish Palach? Some thoughts on the memorialization of Ryszard Siwiec in the Czech-Polish Context. Acta Poloniae Historica, 113, 295-313. https://doi.org/10.12775/APH.2016.113.11

18. Sabatos, C. (1968). The "Burning Body" as an icon of resistance: Literary representations of Jan Palach. Gender and Sexuality in, 2009, 193-217. https://doi.org/10.1057/9780230101203_9

19. Dupont, C., \& Passy, F. (2011). The Arab spring or how to explain those revolutionary episodes? Swiss Political Science Review, 17(4), 447-451. https://doi.org/10.1111/j.1662-6370.2011. 02037.x

20. Zarghami, M. (2012). Selection of person of the year from public health perspective: Promotion of mass clusters of copycat self-immolation. iranian Journal of Psychiatry and Behavioral Sciences, 6(1), 1-11.

21. Hawton, K., \& Williams, K. (2002). Influences of the media on suicide: Researchers, policy makers, and media personnel need to collaborate on guidelines. BMJ, 325(7377), 1374-1375.

22. Time. (2019). Hong Kong's Freedom Protesters Mourn Their Martyr at an Emotional Vigil. https:// time.com/5607928/hong-kong-freedom-protesters-extradition-leung-martyr/. Accessed 10 June 2021.

23. Hollingsworth, J., Shelley, J., \& Coren, A. (2019). How four deaths turned Hong Kong's protest movement dark. https://edition.cnn.com/2019/07/21/asia/hong-kong-deaths-suicide-dark-intl-hnk/ index.html. Accessed 10 June 2021.

24. Robbins, S. (2019). Hong Kong protests: Suicides spark mental health fears. 2019. https://news. sky.com/story/hong-kong-protests-suicides-spark-mental-health-fears-11757909. Accessed 10 June 2021.

25. World Health Organization \& International Association for Suicide Prevention. (2017). Preventing suicide: a resource for media professionals, 2017 update. World Health Organization.

26. Stack, S. (2003). Media coverage as a risk factor in suicide. Journal of Epidemiology and Coтmunity Health, 57(4), 238-240. https://doi.org/10.1136/jech.57.4.238 
27. Fahey, R. A., Matsubayashi, T., \& Ueda, M. (2018). Tracking the Werther Effect on social media: Emotional responses to prominent suicide deaths on twitter and subsequent increases in suicide. Social Science \& Medicine, 219, 19-29. https://doi.org/10.1016/j.socscimed.2018.10.004

28. Au, J. S., et al. (2004). Newspaper reporting of suicide cases in Hong Kong. Crisis, 25(4), 161-168. https://doi.org/10.1027/0227-5910.25.4.161

29. Cheng, Q., et al. (2018). The role of media in preventing student suicides: A Hong Kong experience. Journal of Affective Disorders, 227, 643-648. https://doi.org/10.1016/j.jad.2017.11.007

30. Zeng, X. Y., Chau, P. H., \& Yip, P. S. F. (2019). Improving the monitoring of suicide incidence by estimating the probability of news reporting. Statistics in Medicine, 38(26), 5103-5112. https://doi. org/10.1002/sim.8353

31. Lee, F. (2020). Solidarity in the anti-extradition bill movement in Hong Kong. Critical Asian Studies, 52(1), 18-32. https://doi.org/10.1080/14672715.2020.1700629

32. Kow, Y. M., B. Nardi, B., Cheng, W. K. (2020). Be water: Technologies in the Leaderless AntiELAB Movement in Hong Kong. In: n Proceedings of the 2020 CHI Conference on Human Factors in Computing Systems, p. 1-12. https://doi.org/10.1145/3313831.3376634.

33. Chen, Y. Y., Yang, C. T., Pinkney, E., \& Yip, P. S. F. (2021). Suicide trends varied by age-subgroups during the COVID-19 pandemic in 2020 in Taiwan. Journal of the Formosan Medical Association. https://doi.org/10.1016/j.jfma.2021.09.021.

34. Niederkrotenthaler, T., Voracek, M., Herberth, A., Till, B., Strauss, M., Etzersdorfer, E., \& Sonneck, G. (2010). Papageno v Werther effect. BMJ: British Medical Journal (Online), 341. https://doi.org/ 10.1136/bmj.c5841.

35. Au, C. H., Ho, K. K., \& Chiu, D. K. (2021). The role of online misinformation and fake news in ideological polarization: Barriers, catalysts, and implications. Information Systems Frontiers, pp. 1-24. https://doi.org/10.1007/s10796-021-10133-9.

36. Cheng, Q., et al. (2017). Assessing suicide risk and emotional distress in Chinese social media: A text mining and machine learning study. Journal of Medical Internet Research, 19(7), e243. https:// doi.org/10.2196/jmir.7276

37. Ahmed, S., Jaidka, K., \& Cho, J. (2017). Tweeting India's Nirbhaya protest: A study of emotional dynamics in an online social movement. Social Movement Studies, 16(4), 447-465. https://doi.org/ $10.1080 / 14742837.2016 .1192457$

Publisher's Note Springer Nature remains neutral with regard to jurisdictional claims in published maps and institutional affiliations. 